Arq. Bras. Med. Vet. Zootec., v.66, n.6, p.1681-1686, 2014

\title{
Helicobacteriose em leitões: imuno-histoquímica em amostras colhidas por meio de gastroscopia
}

\author{
[Helicobacter infection in piglets: Immunohistochemical analysis in mucosal \\ samples collected using gastroscopy] \\ R.L. Silveira ${ }^{1}$, A.C.M. Cruz ${ }^{1}$, F.A.G.C. Weber $^{2}$, V.A.N. Degani ${ }^{1}$, \\ E.J. Abílio ${ }^{3}$, E.C.Q. Carvalho ${ }^{3}$ \\ ${ }^{1}$ Universidade Federal Fluminense - Niterói, RJ \\ ${ }^{2}$ Unigranrio - Duque de Caxias, RJ \\ ${ }^{3}$ Universidade Estadual do Norte Fluminense - Campos dos Goytacazes, RJ
}

\begin{abstract}
RESUMO
O diagnóstico da úlcera gastroesofágica (UGE) é fundamental para que se tente a recuperação dos afetados, entretanto há grande dificuldade na sua realização de forma precisa. Modernos endoscópios são ferramentas importantes nesse sentido e podem ser usados para determinar em que fase da criação a ulceração ocorre. A bactéria Helicobacter tem sido associada à UGE e gerado perdas econômicas. Tendo em vista que poucos trabalhos têm sido realizados para identificação do problema precocemente, este estudo teve como objetivo detectar as lesões relacionando-as, ou não, com a bactéria Helicobacter spp., mediante análises macroscópica e histopatológica de amostras colhidas por meio da gastroscopia. Foram utilizados 20 animais de ambos os sexos, pesando entre 22 e 26kg e com 65 dias de idade. O aparelho utilizado foi um gastrovideoscópio da marca Karl Storz, modelo 1380NKS. Foram colhidas amostras das regiões anatômicas aglandular (quadrilátero esofágico) e glandular (cárdica, fúndica e pilórica) para o teste ultrarrápido da urease e para as avaliações histopatológicas e imuno-histoquímicas. Onze animais apresentaram lesões na região aglandular à endoscopia, e microscopicamente 15 animais apresentaram paraqueratose. Em 18 animais, foram observadas alterações em pelo menos uma das três regiões glandulares. As lesões foram maiores na região cárdica, seguida da antral e da fúndica. Em relação ao teste ultrarrápido da urease, sete animais foram negativos nas quatro regiões, e 13 positivos em pelo menos uma delas. Em relação à imuno-histoquímica (IHQ), 10 animais foram negativos em todas as regiões e 10 foram positivos em pelo menos uma delas. Os achados pré-ulcerativos não demonstraram relação com o Helicobacter spp., que apresenta caráter saprofítico e oportunista confirmado pela sua imunomarcação em áreas sem lesão.
\end{abstract}

Palavras-chave: leitão, gastroscopia, Helicobacter spp., imuno-histoquímica

\begin{abstract}
Gastroesophageal ulcer (GEU) diagnosis is fundamental for the treatment and recovery of the affected animal stock. GEU is a condition affecting animals, resulting in depletion of animal stock and subsequent economic losses. Helicobacter spp. have been associated with GEU. Modern endoscopes are important for detecting the stage of the breeding process at which the ulceration occurs. However, few studies regarding early detection of GEU have been conducted. Therefore, we aimed to identify whether GEU lesions were related to Helicobacter spp. infection, using gastroscopy as a diagnostic technique for macroscopic and histopathological analyses. Twenty piglets (both male and female) with a mean age of 65 days were included (weight, 22-26 kg). We used a Karl Storz Gastrovideoscope (model 1380NKS). Samples from nonglandular and glandular (cardia, fundus, and pylorus) regions were collected for the ultra-rapid urease test and for histopathological and immunohistochemical (IHC) evaluations. Eleven
\end{abstract}

Recebido em 23 de setembro de 2013

Aceito em 19 de fevereiro de 2014

E-mail: renatosilveira@vm.uff.br 
animals showed macroscopic lesions in the nonglandular region during endoscopy, and 15 animals showed parakeratosis on histological analysis. Lesions in at least 1 glandular region were observed in 18 animals. The lesions were bigger in the cardiac region, followed by those in the antrum and the fundus. Regarding the ultra-rapid urease test, 7 animals were negative in all 4 regions and 13 were positive in at least 1 region. On IHC, 10 animals were negative in all 4 regions and 10 were positive in at least 1 region. However, pre-ulcerative findings were not correlated with Helicobacter spp. infection in the present study. The positive IHC findings for Helicobacter spp in regions without ulcerative lesions suggest its saprophytic and opportunistic nature.

Keywords: piglet, gastroscopy, Helicobacter spp., immunohistochemistry

\section{INTRODUÇÃo}

O diagnóstico da úlcera gastroesofágica (UGE) se dá por meio da história clínica e de achados de necropsia; dessa forma, as lesões são prontamente observadas e diagnosticadas.

A fim de acompanhar a situação do problema nas granjas, as monitorias patológicas nos matadouros também são uma eficiente ferramenta de avaliação. É o sistema mais utilizado e padronizado no âmbito mundial. A relação entre esses achados patológicos, com base em lesões macroscópicas observadas, e os animais na granja, ainda que limitada na sua especificidade diagnóstica, pode indicar importantes problemas no rebanho (Davies et al., 1995). Entretanto, quando uma enfermidade afeta animais jovens, os resultados obtidos no matadouro com animais em idade de abate não têm estreita relação com a sua ocorrência, devido à dinâmica do processo patológico, o qual tende a reparar-se (Hill et al., 1994).

Para animais com UGE, o diagnóstico precoce é fundamental para que se tente a recuperação dos afetados. Pesquisas na área vêm utilizando modernos endoscópios portáteis como ferramentas práticas no diagnóstico do problema. Eles podem ser usados para determinar a fase específica da produção em que a ulceração ocorre, mas sem o sacrifício de animais (Mackin et al., 1997). Segundo Kopinski et al. (2007), o endoscópio é uma ferramenta viável na avaliação da saúde gastroesofágica.

A bactéria Helicobacter suis tem sido associada à UGE e, uma vez que se encontra difundida na população suína (Grasso et al., 1996), constitui um grande problema (Baele et al., 2008), pois diminui o ganho de peso (De Bruyne et al., 2012) e leva a perdas econômicas (Friendship, 2004).
Tendo em vista que poucos trabalhos têm sido realizados para identificação do problema precocemente, este estudo teve como objetivo detectar lesões relacionando-as, ou não, com a bactéria Helicobacter spp. por meio da análise macroscópica e histopatológica de amostras colhidas por meio da gastroscopia.

\section{MATERIAL E MÉTODOS}

O trabalho, submetido e aprovado ao CEUA $\left(\mathrm{N}^{0} 127\right)$, foi realizado nas dependências do Hospital Veterinário da Universidade Estadual do Norte Fluminense Darcy Ribeiro. Foram utilizados 20 animais, oriundos de granja comercial, de ambos os sexos, pesando entre $22 \mathrm{e}$ $26 \mathrm{~kg}$, com o mesmo manejo alimentar e com 65 dias de idade.

O aparelho utilizado foi um gastrovideoscópio da marca Karl Storz (modelo 1380NKS), com diâmetro distal de inserção de 7,8mm e canal de biópsia de 2,8mm. Seu tubo de inserção possuía $110 \mathrm{~cm}$ de comprimento, e sua extremidade distal apresentava quatro ângulos de deflexão. O cordão universal acoplava-se a uma fonte de luz e ar.

Os animais foram submetidos a jejum alimentar e hídrico de 12 e seis horas, respectivamente. A indução foi realizada com cloridrato de cetamina, com dosagem de $15 \mathrm{mg} / \mathrm{kg} \mathrm{IM}$, e a sedação com midazolan, com dosagem de $0,5 \mathrm{mg} / \mathrm{kg}$ IM. Após todo o procedimento, os animais continuaram em observação até o seu total restabelecimento.

Com o animal em decúbito lateral e com o uso de abre-boca, introduziu-se o tubo de inserção do gastrovideoscópio lubrificado com xilocaína gel. Após inspeção do esôfago, a extremidade distal foi deslocada até a cárdia. Com a passagem por essa região, visualizou-se o estômago de 
forma ampla, verificando-se a conformação, a movimentação e o aspecto da mucosa.

Através do canal de biópsia, foi introduzida uma pinça com a qual se colheram amostras das partes anatômicas aglandular e glandular (cárdica, fúndica e pilórica) para o teste rápido da urease e para as avaliações histopatológicas e imuno-histoquímicas.

Na avaliação endoscópica, utilizou-se o seguinte escore macroscópico para observação da integridade da região aglandular: as lesões ulcerativas foram graduadas de zero a cinco, sendo grau 0 atribuído ao aspecto normal (liso, brilhoso e esbranquiçado); grau 1, àquele com aparência parcialmente rugosa e com alguma área com pigmentação amarelada; grau 2, àqueles com a região totalmente rugosa $\mathrm{e}$ espessada e com áreas de pigmentação amarelada; grau 3, àqueles com a região aglandular totalmente rugosa, espessada e com pigmentação amarelada em toda a sua superfície; o grau 4, àqueles com a região totalmente rugosa, espessada, com pigmentação amarelada em toda a sua superfície e, ainda, mostrando áreas de erosão; e grau 5, àqueles com aparência totalmente rugosa, espessada, com pigmentação amarelada em toda a sua superfície e com áreas de ulceração.

Para o resultado do teste ultrarrápido da urease, utilizou-se o critério de negativo (cor do meio inalterado) e positivo (alteração de cor) nos primeiros cinco minutos.

Para o diagnóstico histopatológico, as amostras colhidas foram colocadas em frascos identificados contendo solução de formalina neutra tamponada a $10 \%$ e encaminhados ao Laboratório de Morfologia e Patologia Animal (LMPA), onde foram processadas. O processamento histotécnico foi por inclusão em parafina para coloração de rotina.

Para realização da técnica de imunohistoquímica, foram feitas seções de $4 \mu \mathrm{m}$ das regiões supracitadas em lâminas silanizadas. Estas, por sua vez, foram desparafinadas em banhos de xilol e reidratadas em banhos de álcool e água deionizada. Em seguida, realizouse o processo para recuperação antigênica com uso do tampão citrato em banho-maria a $96^{\circ} \mathrm{C}$, seguindo para o bloqueio da peroxidase endógena, quando os espécimes foram tratados com solução alcoólica (metanol) de peróxido de hidrogênio a $10 \%$. Após secagem cuidadosa das lâminas, os cortes foram circundados com Dako pen ${ }^{\circledR}$ (Dako, CA, USA) e incubados com solução a $1 \%$ de soroalbumina sérica bovina livre de ácidos graxos e $1 \%$ de leite em pó desnatado diluídos em Tris-NaCL para bloqueio de proteínas inespecíficas. Depois de descartada a solução de bloqueio, as seções foram incubadas com anticorpo policlonal anti-Helicobacter pylori (Rabbit anti-Helicobacter pylori polyclonal antibody, Spring, E3014), diluído 1:100, overnight em câmara úmida. No dia seguinte, as lâminas foram lavadas com solução salina e tratadas com o kit LSAB-HRP ${ }^{+}$(Dako, CA, USA) e reveladas com o kit cromógeno DAB (Dako, CA, USA). Por fim, os espécimes foram contracorados com hematoxilina de Harris, desidratados em banhos de alcoóis e montados com resina sintética. Para análise das lâminas, usou-se a microscopia óptica de luz.

A fim de se analisar a correspondência entre as lesões macroscópicas e microscópicas com os achados imuno-histoquímicos e com os testes de urease, foi realizado o teste de qui-quadrado, considerando-se 5\% de probabilidade de erro.

\section{RESULTADOS E DISCUSSÃO}

Neste trabalho, a idade dos animais foi de 65 dias, mas, em animais comerciais, as úlceras gástricas podem afetar suínos de várias idades, com as maiores taxas acontecendo no intervalo de três a seis meses (Deen, 1993).

Onze animais (55\%) apresentaram lesões na parte aglandular à endoscopia, enquanto nove (45\%) não revelaram alteração. Vários autores, em avaliações macroscópicas, relatam porcentagens acima de $70 \%$ de algum tipo de lesão em seus estudos (Proietti et al., 2010; Swaby e Gregory, 2012) enquanto outros mencionam valores abaixo disso (Dalla Costa et al., 2006).

Dos animais com lesão, sete foram classificados no grau 1, três no grau 2 e um no grau 3 . Neste estudo, o escore foi firmado em razão de a ocorrência de lesões, nessa região, não se apresentar de forma severa, privilegiando, assim, as lesões pré-iniciais ou iniciais não ulcerativas. De forma diferente, outros autores (Yamasaki et 
al., 2006; Swaby e Gregory, 2012) utilizam quatro graus em seus escores, visto que suas pesquisas foram realizadas com animais em idade de abate e com maior incidência de lesões mais graves.

Microscopicamente, 15 animais (75\%) apresentaram paraqueratose. Esses resultados coincidem com os de Marruchella et al. (2004) e se assemelham aos 77,6\% de Yamasaki et al. (2009). Dos animais com paraqueratose, sete mostraram a forma acentuada, quatro a moderada e quatro a discreta.

Comparando as lesões endoscópicas e microscópicas dessa região, verificou-se que, dos nove animais com grau 0, quatro não apresentaram paraqueratose, três apresentaram de forma discreta e dois de forma moderada. Dos sete animais com grau 1, três apresentaram paraqueratose acentuada, dois moderada, um discreta (PM) e um não apresentou. Dos três com grau 2, todos mostraram a forma acentuada. O único animal com grau 3 apresentou a forma acentuada.

$\mathrm{Na}$ parte glandular, em 18 animais, foram observadas alterações em pelo menos uma das três regiões, e somente dois animais não apresentaram alteração em nenhuma das três; em 13, observou-se hiperemia; em cinco, houve formação de catarro; em três deles, houve aumento da atividade mucípara; e, em três deles, houve erosão.

As lesões na parte glandular foram maiores na região cárdica, seguida da antral e da fúndica, mesmo resultado encontrado por Silva et al. (2001). Na região das glândulas cárdicas, cinco animais não apresentaram nenhuma alteração, e 15 apresentaram. Nas regiões das glândulas pilóricas e fúndicas, 10 animais apresentaram alteração, e 10 não.

Em relação ao teste ultrarrápido da urease, nas quatro regiões, sete animais foram negativos, e 13 positivos em pelo menos uma delas, mas nenhum deles foi positivo em todas. Em nenhuma delas foi observada diferença estatística significativa com relação à presença da bactéria.

Ainda sobre o teste ultrarrápido da urease, nas regiões aglandular e das glândulas fúndicas, todos os animais foram negativos. Na região do antro, 11 foram positivos, e nove negativos. $\mathrm{Na}$ região das glândulas cárdicas, cinco foram positivos, e 15 negativos.

O teste ultrarrápido apresenta mais de 90\% de sensibilidade (Calvet et al., 2010), mas pequenas alterações na coloração, mesmo positivas, podem não ser distinguidas do que é considerado normal (McIntosh et al., 2010).

Em relação à imuno-histoquímica (IHQ), 10 animais foram negativos em todas as regiões e 10 foram positivos em pelo menos uma delas, sendo somente um animal positivo em todas. Neste trabalho, não foram observadas diferenças estatísticas significativas com relação à presença da bactéria em nenhuma das regiões.

Na parte aglandular, dois foram positivos, e 18 negativos. $\mathrm{Na}$ região do antro, 10 foram positivos, e 10 negativos. $\mathrm{Na}$ região das glândulas cárdicas, três foram positivos, e 17 negativos. Na região das glândulas fúndicas, dois foram positivos, e 18 negativos.

Esses resultados são concordantes com os encontrados por Szeredi et al. (2005), que, por meio da IHQ, encontraram maior infecção na região cárdica, seguida da pilórica e da fúndica. Uma das vantagens dessa técnica é o diagnóstico de cepas predominantemente cocoides, que, nas colorações de rotina, são mais difíceis de serem observadas (McNulty et al., 2011). Por outro lado, Park et al. (2004), por meio de outras técnicas de diagnóstico, encontraram altas taxas de infecção do Helicobacter, especialmente na região do antro.

Haesebrouck et al. (2009) relataram que o Helicobacter suis está associado às gastrites e úlceras gástricas, e Yamasaki et al. (2009) sugeriram que a bactéria pode ter importante função no estabelecimento de lesões precoces, aumentando a possibilidade de seu agravamento com o avanço da idade.

Ainda segundo Yamasaki et al. (2006), a associação entre Helicobacter spp. e a ocorrência de úlceras gastroesofágicas em suínos tem sido controversa. Embora alguns autores (Szeredi et al., 2005; Proietti et al., 2010) tenham observado a relação entre a presença do Helicobacter spp. e a prevalência e a severidade das úlceras 
gástricas, outros não o fizeram (Ramis et al., 2007).

As possíveis razões para essa inconsistência podem ser a utilização de várias técnicas para determinação da presença da bactéria, cultivos distintos do Helicobacter spp., o que mostra maior ou menor patogenicidade, e o fato de alguns trabalhos serem experimentais enquanto outros observacionais (Taylor e Friendship, 2011).

\section{CONCLUSÕES}

Diante do exposto, pôde-se concluir que os achados pré-ulcerativos não demonstraram relação com o Helicobacter spp., que apresenta caráter saprofítico e oportunista confirmado pela sua imunomarcação em áreas sem lesão.

\section{REFERÊNCIAS}

BAELE, M.; DECOSTERE, A.; VANDAMME, L. et al. Isolation and characterization of Helicobacter suis sp. nov. from pig stomachs. Int. J. Syst. Bacteriol. v.58, p.1350-1358, 2008.

CALVET, X.; LEHOURS, P.; LARIO, S.; MÉGRAUD, F. Diagnosis of Helicobacter pylori infection. Helicobacter, v.15, Supl. 1, p.7-13, 2010.

DALlA COSTA, O.A.; COLDEBELlA, A.; COSTA, M.J.R.P. et al. Período de descanso dos suínos no frigorífico e seu impacto na perda de peso corporal e características do estômago. Cienc. Rural, v.36, p.1582-1588, 2006.

DAVIES, P.R.; BAHNSON, P.B.; GRASS, J.J. et al. Comparison of methods for measurement of enzootic pneumonia lesions in pigs. Am. J. Vet. Res., v.56, p.709-714, 1995.

DEEN, J. The problem of gastroesophageal ulcers: A field description. In: ALLEN D. LEMAN SWINE CONFERENCE, , 1993, Saint Paul, Minnesota-USA. Proceedings. Saint Paul, 1993 p.137-138.

De BRUYNE E.; FLAHOU B.; CHIERS K. et al. An experimental Helicobacter suis infection causes gastritis and reduced daily weight gain in pigs. Vet. Microbiol., v.160, p.449-454, 2012.

FRIENDSHIP, R.M. Gastric ulceration in swine. J. Swine Health Prod, v.12, p.34-35, 2004.
GRASSO, G.M.; RIPABELLI, G.; SAMMARCO, M.L. et al. Prevalence of Helicobacter-like organisms in porcine gastric mucosa: A study of swine slaughtered in Italy. Comp. Immunol., Microbiol. and Infect. Dise.. v.19, p.213-217, 1996.

HAESEBROUCK, F.; PASMANS, F.; FLAHOU, B. et al. Gastric Helicobacters in domestic animals and nonhuman primates and their significance for human health. Clin. Microbiol. Rev., v.22, p.202-223, 2009.

HILL, M.A.; SCHEIDT, A.B.; TECLAW, R.F. et al. Relationship between the indicators of performance and the weight of pneumonic lesions from pigs at slaughter. Res. Vet. Sci., v.56, p.240-244, 1994.

KOPINSKI, J.S.; FOGARTY, R.; McVEIGH, J. Effect of s-methylmethionine sulphonium chloride on oesophagogastric ulcers in pigs. Aust. Vet. J., v.85, p.363-367, 2007.

MACKIN, A.J.; AYLES, H.L.; FRIENDSHIP, R.M. et al. Development and evaluation of endoscopic technique permitting rapid visualization of the cardiac region of porcine stomach. Can. J. Vet. Res. v.61, p.121-127, 1997.

MARRUCHELLA, G.; DI LEONARDO, M.; DI GUARDO, G. et al. Heat shock proteins (HSPs) 27, 72 and 73 in normal and pré-ulcerative mucosa of the gastric pars oesophagea in swine. J. Comp. Pathol. v.131, p.10-17, 2004.

McINTOSH, K.A.; KRAKOWKA, S.; RINGLER, S.S.; ELLIS, J.A. In situ detection of ureasepositive Helicobacter pylori-like organisms on swine gastric mucosa. Can. J. Vet. Res. v.74, p.237-240, 2010.

McNULTY, C.A.M.; LEHOURS, P.; MÉGRAUD, F. Diagnosis of Helicobacter pylori infection. Helicobacter. v.16, sup.1, p.10-18, 2011.

PARK, J.H.; SEOK, S.H.; CHO, S.A. et al. The high prevalence of Helicobacter spp. in porcine pyloric mucosa and its histopathological and molecular characteristics. Vet. Microbiol., v.104, p.219-225, 2004.

PROIETTI, P.C.; BIETTA, A.; LEPRI, E. et al. Detection of Helicobacter spp. in gastric, fecal and saliva samples from swine affected by gastric ulceration. J. Vet. Sci., v.11, p.221-225, 2010. 
RAMIS, G.; GÓMEZ, S.; PALLARÉS, F.J.; MUÑOZ, A. Prevalence of Helicobacter-Like bactéria in the gastric mucosa of pigs slaughtered in south-east Spain. An. Vet. Murcia., v.23, p.7586, 2007.

SILVA, J.C.P.; SANTOS, J.L.; BARBOSA, A.J.A. Gastrite em suínos: frequência, relação com a úlcera gástrica e com a densidade de células endócrinas do estômago. Rev. Bras. Cienc. Vet., v.8, p.40-43, 2001.

SWABY, H.; GREGORY, N.G. A note on frequency of gastric ulcers detected during post mortem examination at a pig abattoir. Meat Sci., v.90, p.269-271, 2012.

SZEREDI, L.; PALKOVICS, G.; SOLYMOSI, N. et al. Study on the role of gastric Helicobacter infection on gross pathological and histological lesions of the stomach in finishing pigs. Acta Vet. Hung., v.53, p.371-383, 2005.
TAYLOR, S.; FRIENDSHIP, R.M. A systematic review of the association between Helicobacter spp. and the development of gastric ulcers in swine. In: ANNUAL MEETING OF AMERICAN ASSOCIATION OF SWINE VETERINARIANS. 11., 2011. Proceedings... Phoenix, Arizona-USA. p.337-338, 2011.

YAMASAKI, L.; ASSIS, M.S.; ROSSETO, V.J.V. Bracarense, A.N.F.R.L. Lesões gástricas em suínos: Ocorrência e relação com o gênero, peso ao abate e presença de Helicobacter spp. Semina: Cienc. Agrárias, v.27, p.463-470, 2006.

YAMASAKI, L.; BOSELLI-GROTTI, C.C.; ALFIERI, A.A. et al. Alterações histológicas da pars esophagea de suínos e sua relação com Helicobacter spp. Arq. Bras. Med. Vet. Zootec., v.61, p.553-560, 2009. 